\title{
Designing interaction in digital tabletop games to support collaborative learning in children
}

-Author information removed for anonymous reviewing-

\begin{abstract}
According to Dillenbourg et al (1996) collaborative learning occurs when two or more people try to learn something together. This process consists of four successive stages, one of which concerns collaborative interactions. In this paper we present two studies that implemented two different ways of increasing the number of collaborative interactions.

To increase the number of collaborative interactions in a game, the first study focused on degrees of collaboration (Kahn and Mentzer, 1996) and the second study focused on cooperative gestures (Morris et al, 2006), which were used in the third degree of the first study. In order to facilitate collaborative interactions and its properties (Dillenbourg, 1991), we decided to design two digital tabletop games with tangible interaction that both require collaboration to win.

The evaluations in both studies, by means of the Wizard of $\mathrm{Oz}$ method, showed a significant increase in collaborative interactions. We also found that verbal and gestural interactions are a better measure criterion for tabletop games than how much people look at eachother.
\end{abstract}

\section{Keywords}

Collaborative learning, cooperative gestures, social interaction, digital tabletop games, tangible interaction, interaction design.

\section{Introduction}

Learning in groups, in particular collaborative learning; is considered an effective way of learning (Dillenbourg et al, 1996). Therefore many schools offer learning activities in groups. When children are collaborating with each other, they work on their social skills, achieve better results and their critical thinking is enhanced (Gokhale, 1995). For example, when a group of school children is making a medieval castle together; they may discuss the appearance of the castle, read and exchange information about the Middle Ages, point to issues that need attention and make decisions on the decoration. Summarizing, during collaborative learning activities children develop knowledge as well as social skills.

In this paper we focus on designing collaborative interactions for children and we present two studies. The first study is focused on increasing the social interaction during collaboration by means of different levels in the game. By using Kahn's and Mentzer (1996) degrees of collaboration, the children are stimulated to work together more intensively by reaching new levels in the game. The evaluation of the game, using the Wizard of Oz method, showed significant increase in collaborative interactions.

In the second study the focus is also on designing collaborative interactions for children, this time on improving social skills by using cooperative gestures. Cooperative gestures are interactions where the system interprets the gestures of more than one user as contributing to a single, combined command' (Morris et al, 2006), e.g. two people pulling the corners of a photo to enlarge it. A property of cooperative gestures is the execution by multiple users, e.g. the users have to work together to fulfill a task. In the first study cooperative gestures were integrated in the highest game level, which lead to a significant increase of collaborative interactions. Therefore, in the second study cooperative gestures became the focus. By using cooperative gestures in a digital tabletop multiplayer game, children have to work together to complete the game. In this study the cooperative gestures are formed by a set of dedicated tangible artifacts designed for the game.

The theoretical background section (Section 2) will explain the key topics presented in this paper: collaborative learning, tangible interaction and digital tabletops and social interaction. Sections 3 and 4 will 
cover both case studies and Sections 5 and 6 will conclude the paper with a general discussion and conclusions.

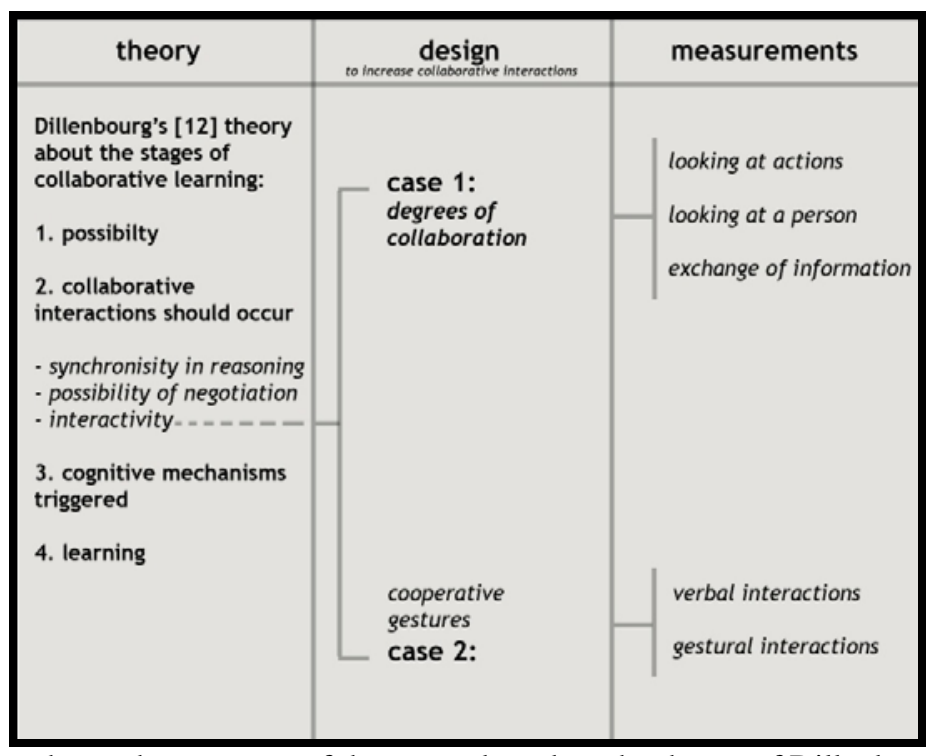

Figure 1 This figure shows the structure of the paper, based on the theory of Dillenbourg et al (1996). We aimed to increase collaborative learning by focusing on collaborative interactions. In study 1 the degrees of collaboration have been designed to increase the collaborative interactions; in study 2 cooperative gestures have been designed to do so. In study 1 three properties were measured to determine whether the collaborative interactions increase; in study 2 this was changed to two properties.

\section{Theoretical Background}

\subsection{Collaborative Learning}

Dillenbourg et al (1996) defines collaborative learning as: 'a situation in which two or more people learn or attempt to learn something together'. Collaborative learning can occur at schools, at playgrounds and even at work. Roschelle and Teasley (1995) support this theory of collaborative learning by stating that collaborative learning involves the mutual engagement, e.g. the attention of all participants, in a coordinated effort to solve the problem together. Moreover, as Baker et al. (1999) argue; in order to collaboratively learn, some form of mutual understanding is needed; the so-called grounding. They describe two levels of grounding: pragmatic grounding and semantic grounding. Pragmatic grounding is part of learning to collaborate, while semantic grounding relates to attaining a mutual understanding of what is meant, it thus relates closely to learning in a certain knowledge domain. Related to the argumentation of grounding is the theory of Stahl, Koschmann and Suthers (2006). They state that collaboration is primarily conceptualized as a process of shared meaning making. Achieving collaborative learning involves phenomena like the negotiation and sharing of meaning.

In our study we support the theory of Dillenbourg et al (1996). In his work he describes collaborative learning as follows: Collaborative learning occurs according to the following successive stages: 1) a situation should occur in which collaborative learning is possible, 2) collaborative interaction should take place between two or more people, 3) cognitive mechanisms should be triggered and 4) the ultimate effect would be learning. In the study of Gokhale (1995) a cause and effect relation between collaborative interactions and learning can be found. In this study students worked together to achieve a common academic goal. However, the students that fulfilled the task by solely email conversations learned less than those who actively discussed their findings within the group. This can be explained by the fact that the exchange of collaborative interactions is lower in the group of students that communicated via email, which would suggest that collaborative interactions are especially useful to increase learning. To increase the probability of cognitive mechanisms being triggered, thus the probability of learning, the amount of collaborative interactions should be increased. Collaborative interactions have three properties: synchronicity in reasoning, the possibility of negotiation and interactivity (Dillenbourg, 1991). With synchronicity in reasoning is meant the feeling of simultaneous reasoning that people have; for example 
attacking a problem in the same way, with the same mind set. The possibility of negotiation can be seen as the possibility to defend a point of view. By interactivity the extent to which the interactions influence the peers' cognitive processes is meant.

Several ways of measuring these collaborative interactions have been proposed. In the study performed by Hornecker et al (2008) they used awareness indicators to measure successful collaboration. Their indicators were divided in three groups: negative awareness indicators (e.g. interference), positive awareness indicators (e.g. parallel work) and awareness work (e.g. exaggerated movements). In their study these indicators were used to evaluate cooperative work; people working together on a task. However, in our study we aimed at collaborative work, e.g. discussing next steps and no parallel work.

In the work of Fleck et al (2009) the Collaborative Learning Mechanisms framework is presented. The framework discusses the verbal and physical aspects of "Making and accepting suggestions", "Negotiating” and "Maintaining joint attention and awareness". However, this work is also directed towards performing a task. Moreover, their framework has not been evaluated with other tabletop configurations, such as turn taking and joint actions (Fleck et al, 2009).

Therefore, we used the theories of Dillenbourg et al (1996) and Bauminger, Gal and Goren-Bar (2007) to determine the measurement methods in the first study; we measured the information exchange between children and how much they looked at another player or their actions. After evaluating the first study, the measurements in the second study were purely based on Dillenbourg et al (1996). Here we measured the 'proposals for action', 'reactions on these proposals' and 'reasoning in general.

In the first study presented in this paper we aimed for an increase in collaborative interactions by using degrees of collaboration. The term degrees of collaboration is based on the work of Kahn and Mentzer (1996), these degrees are based on their experiences with collaboration levels between departments within the same company.

In the second study presented in this paper we aimed to use a method to increase the amount of collaborative interactions, namely cooperative gestures. The exploration of cooperative gestures initially started from the wish to prevent conflicts over digital artifacts between people when collaborating around a digital tabletop (Greenberg and Marwood, 2004; Hornecker et al, 2008; Morris et al, 2004; Scott, Grant and Mandryk, 2003; Smith, Hixon and Horan, 1998). As a result coordination policies were made and tested in different applications (Magerkurth et al, 2004; Morris et al, 2006; Morris et al, 2004; Ryall et al, 2005). However a group of researchers felt that other interaction techniques could potentially mitigate undesired aspects of group behavior by increasing group awareness of important interactions and encouraging a sense of involvement and togetherness (Morris et al, 2006). This line of thinking led to the concept of cooperative gestures (Morris et al, 2006).

Collaborative and competitive settings encourage people to socially interact with one another. Using competition and collaboration in a game could be a way to encourage children to participate in a social situation. Al Mahmud et al (2007) created the game aMAZEd in which children work in pairs through three different gaming levels against others. They combined collaboration and competition in one game. Suh (2006) state that the need to collaborate is determined by the complexity of the task, the people that are required to fulfill the task and the need to not fail (Suh, 2006). Integrating these requirements in a game setting could be a way to facilitate social interaction.

\subsection{Tangible Interaction \& Digital Tabletops}

As we explained in the introduction we used physical artifacts in the games to support collaboration and in particular realize cooperative gestures. The idea of physical artifacts serving as controls and representations of digital functionality is what defines tangible interaction (Fitzmaurice, Ishii and Buxton, 1995; Ullmer and Ishii, 2000). These physical artifacts bridge and combine the physical and digital world and afford a much richer interaction concerning human senses and skills than traditional graphical user interfaces (GUI) do (Ullmer and Ishii, 2000). In order to be able to use tangible interaction in our games we decided to use a digital tabletop as platform.

A digital tabletop is an interactive table with an embedded display capable of real-time tracking of fingers of multiple users and physical artifacts (Mazalek, 2005). These digital tabletops can support group interaction by allowing face-to-face interaction and multiple inputs from a group of users (for an overview 
of these technologies, see (Mazalek, 2005)). Stewart, Bederson and Druin (1999) was one of the first to use a shared display used by multiple users in a collaborative setting. Their results showed that children preferred the collaborative setting over the traditional individual setting. A collaborative setting results in a higher level of common ground between participants and supports effective collaboration (Tse, Greenberg and Shen, 2006). Another benefit of the tabletop technology is the way digital and physical gaming can be combined. Adding physical handling to digital games creates more space for social interaction. Moreover, games on a digital tabletop can be modified to gain a child's interest and adjusted to a child's skill level. This way the child is more likely to be motivated to play the game and work on its social skills.

More specifically, digital tabletop technology in combination with tangible interaction was chosen for the facilitation and support of cooperative gestures. The visual output of the digital tabletop in combination with the execution of commands in a physical manner is the most suitable way of creating the cooperative gestures.

Several existing games incorporate tangible interaction and digital tabletops e.g., False Prophets (Mandryk, Maranan and Inkpen, 2002), Weathergods (Bakker et al, 2007), KnightMage (Magerkurth et al, 2004) and the STARS adaptation of Monopoly (Magerkurth et al, 2004). None of these games were developed for facilitating collaborative learning, which we intend to do in this paper, but show the advantage of using physical artifacts with a digital platform. Some examples of games have been found in the field of autism to facilitate collaborative learning. Games, such as StoryTable (Bauminger, Gal and Goren-Bar, 2007) and SIDES (Piper et al, 2006), especially designed for High Functioning Autistic children, support a collaborative environment. The StoryTable (Bauminger, Gal and Goren-Bar, 2007) allows children to create stories, while sharing experiences, on a digital tabletop by playing with ladybugs wandering over the screen. Their results suggest that a multi-user interface has potential for enhancing social behaviors and some core language skills. Piper et al (2006) developed SIDES to give HFA children the opportunity to improve their group work skills. Their evaluation shows that SIDES can be used to help the children develop group work skills and get more comfortable in participating in social interaction, e.g. improve their social skills.

\section{Study 1: Degrees of Collaboration}

In the first study we want to stimulate children to work together on a digital tabletop game to increase their social skills. Traditional board games are an example of how social skills can be enhanced by playing games; these games are used in therapies for children that have impairments in social interaction. By combining a traditional board game with the excitement of a computer, children could benefit from this digital multiplayer tabletop game. Our goal is to stimulate children to socially interact with others via a digital tabletop game. We aim to achieve this by applying "degrees of collaboration", which is hypothesized to increase the amount of collaborative interactions and thus social interaction.

\subsection{Game Design}

The next step was to design and develop a game that increases social interaction.

The idea generation phase consisted of a brainstorm session and idea generation sessions, focusing on various aspects of the game. Based on these sessions and literature 24 requirements were constructed, including aspects such as simple and clear dialogues, hand-sized usable objects and clear controllable game play. Approximately 50 ideas were generated and combined into a set of 15 ideas that were evaluated against the requirements. From these ideas five concepts were developed and eventually the concept which fitted the requirements best, Totti, was selected to work with and developed into a game.

The goal of the Totti game is to help a hero go from village to village to gather pieces of a totem pole and build it up. For the setting of the final game narrative we chose North America. The game story is about an Indian orphan, who has to reunite several villages in order to defeat the darkness (evil component). By visiting every village, finding the pieces of the totem pole and building it up, the darkness can be defeated. To support the collaborative process each child plays one of the four gods, representing an element, which guide the hero. The operational rules of the game are especially designed to fit the user group and support the degrees of collaboration implemented in the game. 


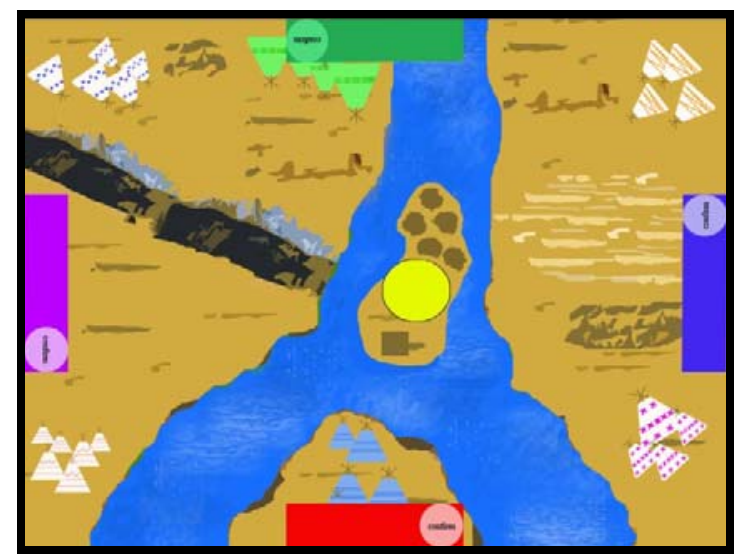

Figure 2. Screenshot of the Totti game concept.

We chose a turn taking game, because it supports a structured way of playing. Moreover, each child gets the opportunity to be part of the game play. Each turn a device with dice function has to be placed on a certain spot on the field from which a number of possible paths is generated. The children discuss the most suitable path and move the hero accordingly. When the hero needs to cross an obstacle he needs an object from one of the gods. For example, when the hero wants to cross the river, he needs the boat from the god of water. Which object has to be used, should be discussed by the children. When they can not come up with a solution the computer will give them a hint. When the hero enters a village, the people of the village send him a request. If completed correctly the hero gets a part of the totem pole. The children can help the hero by combining the powers of the gods and placing them in the temple. For example, the village needs rain because it has been dry for several months. In that case the power air and water have to be combined. The children will need to use two separate objects to execute one command from the computer, this can be seen as a collaborative gesture. Together the children have to decide when a turn is over, and place their god on the 'confirm' tile, when this is not done, the game will not continue. By doing so, each child agrees with the previous actions, which is important for the social aspect of the game. Moreover, it stimulates the children to keep paying attention to the game.

The design was inspired by other tabletop games, namely SIDES (Piper et al, 2006), Weathergods (Bakker et al, 2007) and Storytable (Bauminger, Gal and Goren-Bar, 2007). Specifically, some game mechanics from SIDES (Piper et al, 2006) were used; the confirmation after a turn and the involvement of personal objects. Also the way of designing the game pieces from Weathergods (Bakker et al, 2007) was used and the way of involving children in the game experience by shared interactions from Storytable (Bauminger, Gal and Goren-Bar, 2007).

A set of tangible artifacts was designed (Heijboer and van den Hoven, 2008) to function as game pieces for the game. Every player has his own game pieces, as seen in Figure 3. One representing a god (water, air, fire of earth), and another artifact representing equipment, for example the god of water has a boat-object. The hero is represented as a smaller white figure. For the totem pole the pieces were designed to fit together in random order, by doing so the players are unable to predict how to build up the totem pole.

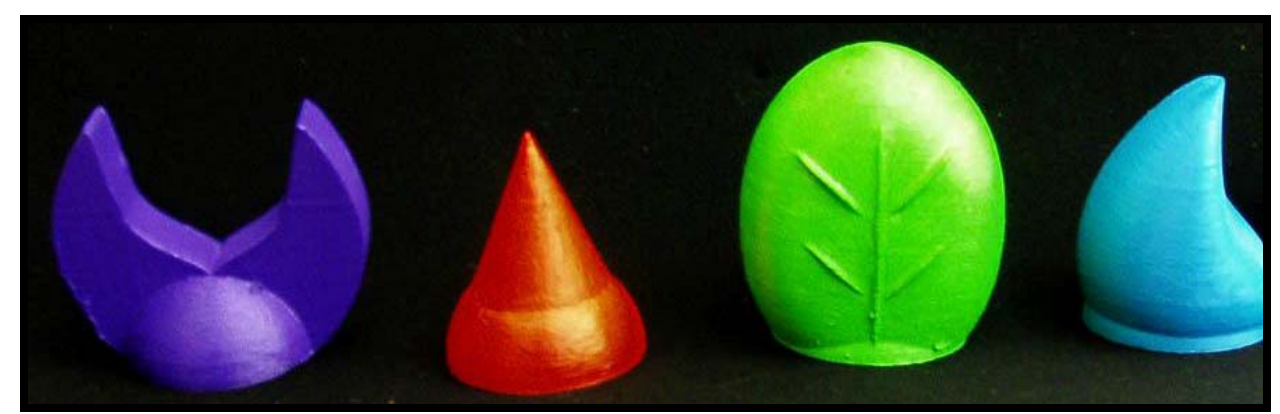

Figure 3: The tangible game artifacts of the Totti game. 
In the game different actions are designed in such a way that they support collaboration . Some actions stimulate the children to pay attention and discuss various options in the game, such as the popping up of a request or the confirmation after a turn. To show the collaboration the objects give haptic feedback; the god objects literally click together. The various actions and the amount of social interaction and collaboration that is needed are divided into separate levels in the game, see Figure 4. The first level includes the tasks; confirming and walking paths. In this level the children need a small amount of collaboration to succeed. Level two includes the tasks of overcoming obstacles and exchanging of objects. In this level the children need to discuss which tools to use and they need each other's objects, thus more collaboration is needed compared to level one. In the third and last level the children need to fulfill a request by the village, which stimulates them to work together to succeed. In this game the highest level of collaboration is required to fulfil the overall game goal successfully. In this highest level, the children need to argue and discuss what to do to fulfill the request, and they have to combine objects to complete the request, e.g. the children need to use cooperative gestures.

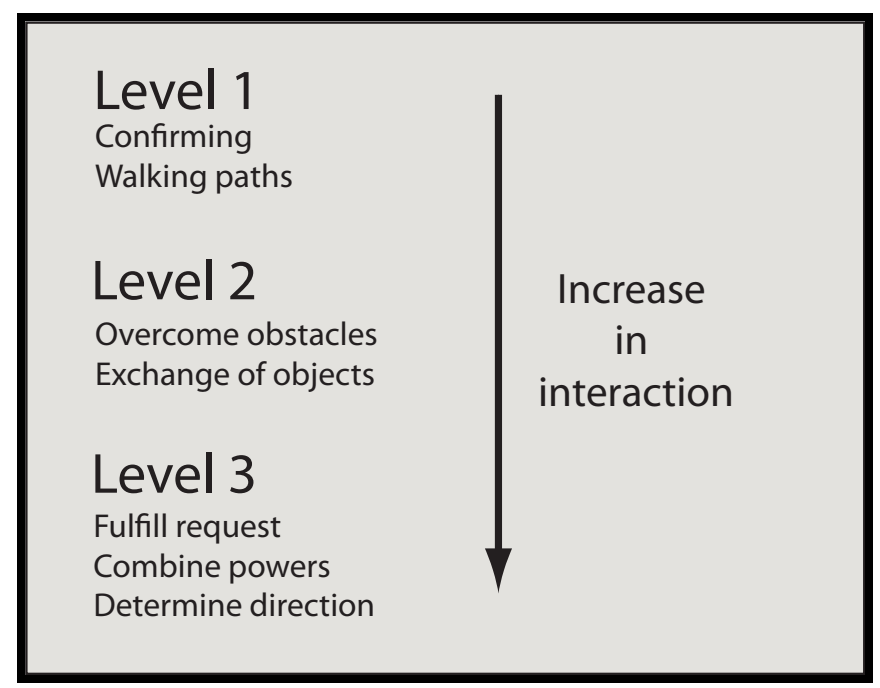

Figure 4: Scheme showing the three different game levels in the Totti game that require an increase in interaction between the players, as seen in the examples of interactions, such as 'confirming' and 'exchange of objects'.

By conducting tests with a paper prototype, as shown in Figure 5, we aimed to find out if the rules were understandable and what needed to be changed. The first pre-test was conducted with the research team to make some initial changes. The changes included rules as turn taking, confirming and entry of gods. After the changes had been processed the game was tested for the second time with five researchers, which were able to play the game as it would be played by children.

The comments and feedback were used to create the final game. The changes included removing the point system, shortening the confirm action and a clear function for the bandit. Next to that the suggestion was given to have a high amount of digital feedback. 


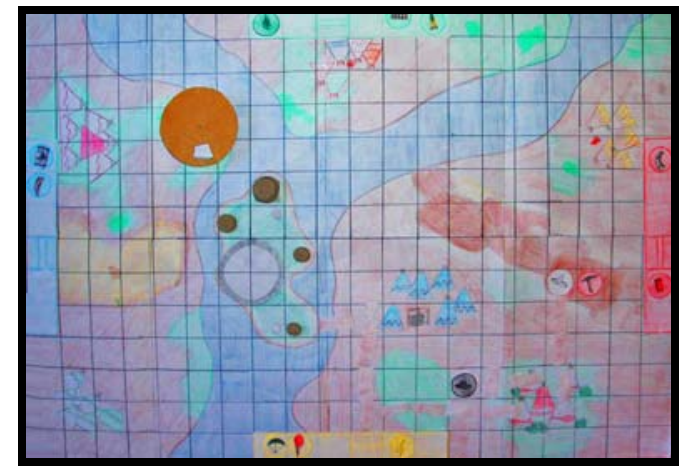

Figure 5: Paper prototype of the Totti concept, without the tangible artifacts.

\subsection{Technical Implementation}

The digital tabletop is based on the technology used in the reacTable (Jorda et al, 2005) which can be programmed by using the ReacTIVision Software (Kaltenbrunner and Bencina, 2007). The hardware consists of two different parts; an infrared camera and a projector. The infrared camera captures finger touch and fiducials. Fiducials are visual markers which can be placed on the bottom of any physical artifacts (Barry and Pitt, 2006), allowing designers to use any object to work with. Based on the interaction with the digital tabletop, the resulting image is projected via a mirror on the horizontal screen of the tabletop (Figure 6).
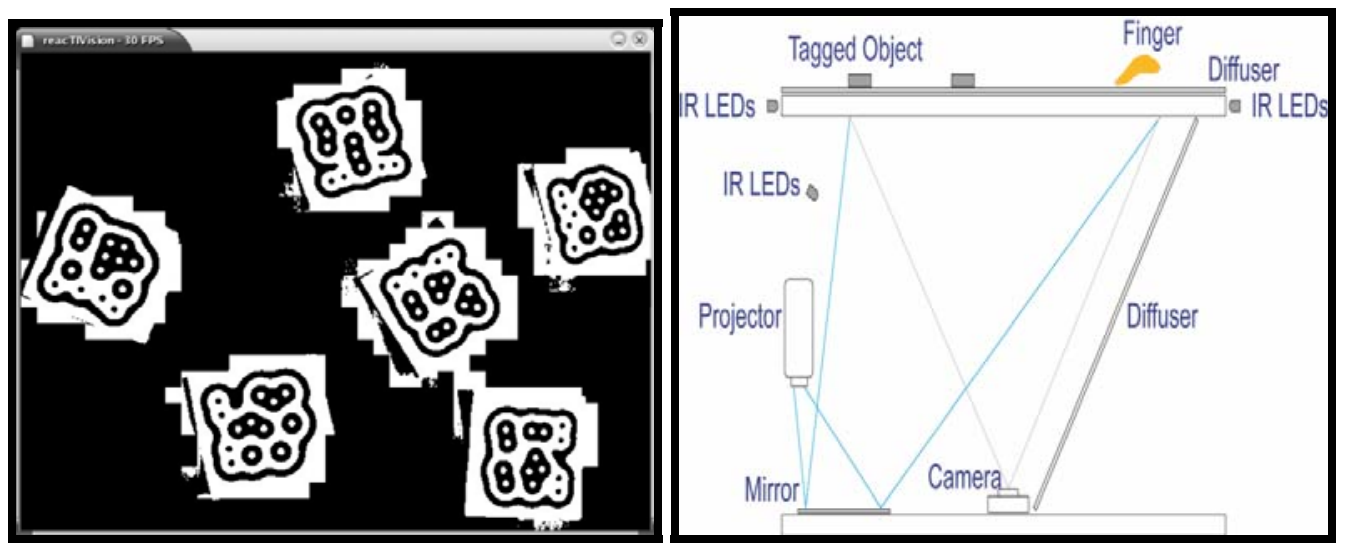

Figure 6: Example of fiducial markers and a schematic overview of the digital tabletop.

The reacTIVision platform can be used with different programs; in this case Processing was chosen. This program allows object oriented programming and supports visual output which was preferred to simulate a board game.

Unfortunately the integration of the hardware and software was problematic. The combination of the computer vision of the table, using the Processing language, which had to be executed on top of the Java platform and a visually heavy program made the game too slow to be playable on the table. However, the game worked correctly on the simulator that was delivered together with the software. This simulator is meant to check the code while working, so it can be assumed that if the hardware would work correctly, the game would also. The problem of the integration of the software and the hardware could have been overcome by more processing power and an upgrade of the other equipment, however, due to time and cost limitation the authors have chosen to evaluate the game by using the simulator provided by reacTIVision. During the comparative study with the children, the game was projected on the table, but the computer vision part was simulated by the experimenter in a wizard-of-oz like manner. This meant that the children played the game while the experimenter copied their actions on a computer. The visual feedback worked as it would normally do. 


\subsection{Methodology}

After the game was implemented, it was evaluated with a group of middle school children, 12 boys and 8 girls in the ages 10 - 13 from a science club, who participated in the sessions. The average age was 11,95. Two girls were in grade 8 , the others were grade 7 . The children all had some gaming experience, varying from playing a game twice per month to approximately twice a week.

We wanted to measure whether the amount of social interaction increases over the different levels, which was the focus of our study. In addition we wanted to find out whether the game appeals to children and if it is fun to play. The parents from each child that participated signed a form of consent and every child signed a form of assent. In five separate sessions children from the science club (STEMCELL) visited us at Georgia Institute of Technology and participated in groups of four. Each evaluation took approximately 20 minutes, an introductory explanation of five minutes and 15 minutes for the gaming itself. In each session the children experienced three levels of social interaction in the game. During the session the participants were being filmed while playing the game. The cameras were focused on the children and not on the game. The children knew that they were being filmed, their parents agreed with this.

The behavior was analyzed based on Bauminger's criteria (Bauminger, Gal and Goren-Bar, 2007) and Dillenbourgs theory (Dillenbourg, 1991) and addresses three different questions:

- How much information did each child exchange with other player?

- How much did each child look at another player?

- How much did each child look at other players' actions?

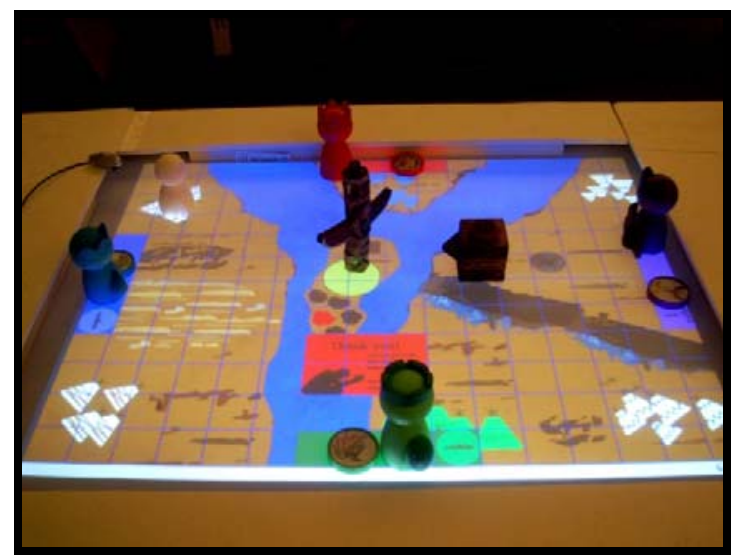

Figure 7: The implemented game Totti.

Each of these criteria was measured per level of collaboration to determine whether the amount of interaction increased. The children were being videotaped, after signing a form of consent, while playing the game. This video was only used to measure the criteria. When counting how much one child looked at another player only reactions were scored, since a reaction is a sign of social behaviour. So when a child was just looking around, this was not scored, since this has nothing to do with social interaction. The same counts for the information criteria; only the information directed to others, involving the game was scored. The numbers used are the amount of times people looked at another player per minute (as a reaction on an action) or looked at another player's action and the times information (a sentence counts as one information exchange) was exchanged per minute. Per game level the interactions were scored per minute per child, therefore when a game was played with four children, the videotape was scored four times, once for each participant.

\section{4 Results Study 1: Degrees of Collaboration}

Overall in the game, we observed that all types of interaction increased when reaching higher levels e.g. levels in which more collaboration is needed. In Figure 8 the quantitative results can be found. The results were divided in three different categories: Information, Person and Action, representing the three main questions in evaluation. As can be seen for every criterion, the differences between level 1 and level 2 are larger than the differences between level 2 and level 3 . With the results a two-sided paired samples t-test (p $<0.05$ ) was done. Table 1 shows the results of this test. The difference in the amount of information 
exchange, looking at another person and looking at another person's action between level 1 to level 2 were in the hypothesized direction all significantly ( $<<0.001, \mathrm{p}<0.05, \mathrm{p}<0.001)$. From level 2 to level 3 the difference in how much information was exchanged increased significantly $(\mathrm{p}<0.01)$, the other two did not increase significantly ( $p>0.05$ and $p>0.05$ ).

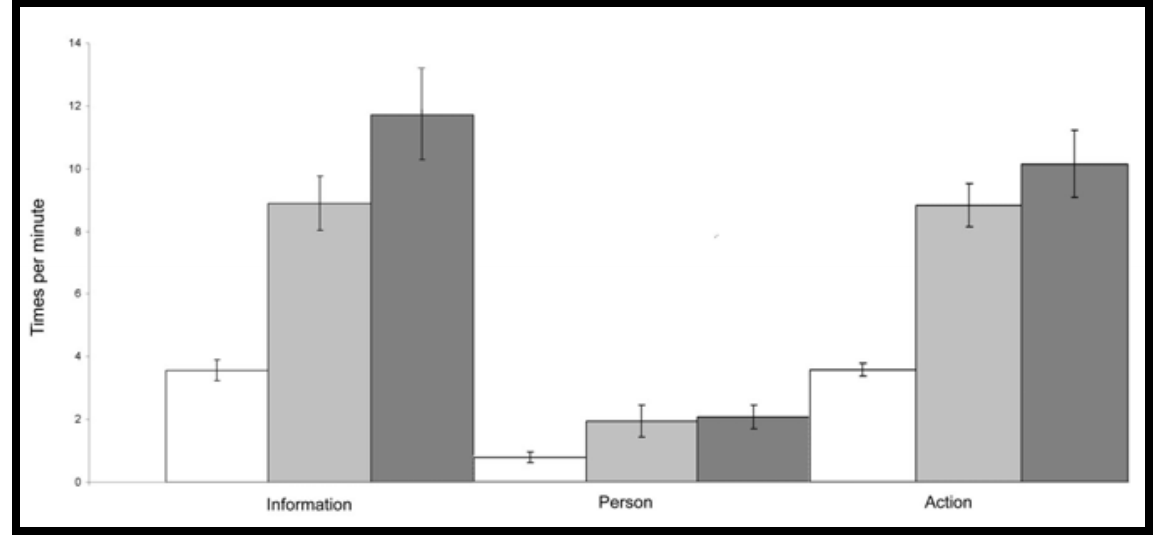

Figure 8: The number of times each child exchanged information, looked at another person and his actions per minute. Represented for all three levels of social interaction embedded in the game (level 1 = white bar, 2 = light grey bar, 3 = dark grey bar).

The amount of looking at each other is very low and does not change a lot over the different levels. This can be explained by the fact that we are using a digital tabletop game on which all the action happens. The consequence of this is that the children only interacted via language and actions and had little eye contact.

Table 1. Comparing social interaction between level 1 and 2 and level 2 and 3 (S means significant and NS means not significant).

Summarizing, this study describes the design of a tangible game on a digital tabletop with the goal to increase social interaction among children. We incorporated three levels of collaboration in the game, in which the children were stimulated to collaborate more per level. Results from the test with 20 children show an increase of collaborative interactions, and thus social interaction. The difference between the first and the second level shows a significant increase in social interaction for all three criteria, this is due to the embedded object exchange and the construction of the assignments, compelling the children to talk to each other. The difference between the second and the third level shows a significant increase in social interaction for the information exchange, this is due to the usage of cooperative gestures; coercing the children to argue and discuss how to complete the assignment, e.g. to collaborate.

\section{Study 2: Cooperative Gestures}

In the second study we present Flourishing Future. The goal of this study is to increase the amount of collaborative interactions by using cooperative gestures. In the first study cooperative gestures were integrated in the highest game level; the children needed multiple (personal) objects to execute one single command. As can be seen in the results of the first study, the third level shows the most promising results and therefore cooperative gestures where the focus in the second study in which the game "Flourishing Future" was designed. To check whether the increase of collaborative interactions is actually caused by the cooperative gestures, two versions of the game where made, one with cooperative gestures and one without. 


\subsection{Game Design}

The goal of the Flourishing Future game is to make a city as environmentally friendly as possible. Figure 10 shows a screenshot of the game. The city can change positively or negatively according to the digital objects the players place in it. An example is building a tree, or making a train station. Each player has its own unique set of digital artifacts that are shown in the personal space on the border of the display (see Figure 9). Thus the children need to debate about which digital artifacts to use to reach the overall goal.

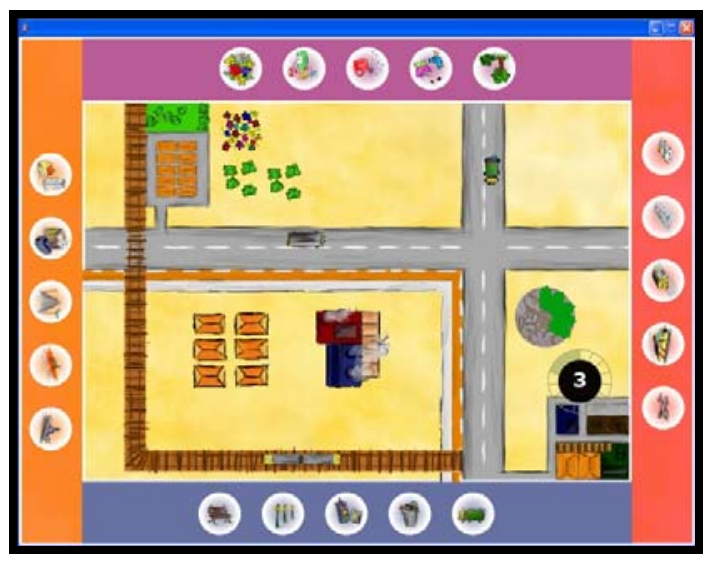

Figure 9: Screenshot Flourishing Future

Reaching a higher level of environmental friendliness is visualized by a tree getting more leaves. This game fits into the genre of Real-Time Strategy gaming (RTS) (Real- Time Strategy Games, 2011). Examples of this genre are Simcity and Rollercoaster Tycoon. Moreover, the game design was inspired by the work of Morris et al (2006) and the Storytable (Bauminger, Gal and Goren-Bar, 2007). We developed two versions of the game, only differing in interaction style: one 'normal' version and one supporting 'cooperative' gestures (see Figure 10). The structure of the game is equal in both versions, only the interaction style differs. In the 'normal' version four tools are used to perform actions. A stamp is used to copy a digital object from the personal space onto the board. The eraser can remove these objects from the board. A stepcounter is used for digitally displaying the steps left. The level the children reached in the game is indicated by the amount of leaves on the tree. In the 'cooperative' version of the game the function of the stepcounter and tree is the same. The stamp and eraser were divided in four pieces, which needed to be combined to perform an action. Each player got one piece of the eraser and stamp, both in the same color. These two cooperative gestures were named combined stamping/erasing.
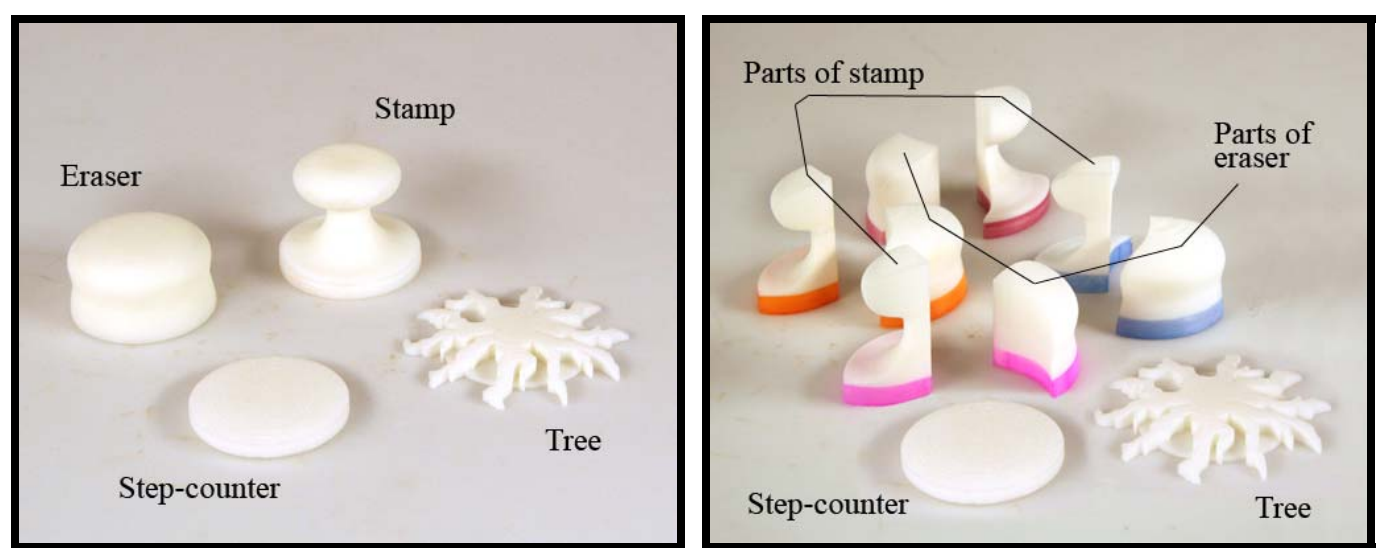

Figure 10: The left picture shows a set of artifacts that can be used individually; the right picture shows a set of artifacts that support cooperative gestures. 
A pilot evaluation was done by a walkthrough using the paper prototype to explain different phases in the game. It was conducted with three researchers. As a result the introduction was shortened and rephrased in a more positive way. The tools and their function were adapted to make them usable for every player in the game. After this first iterative step a second evaluation, with the improved paper prototype, was setup (see Figure 11). Four other researchers, who were not familiar with the game, played it while we applied a wizard-of-oz principle by faking the interaction of the game. The most important result was a lack of (realtime) feedback, but this was planned to be included in the digital version of the game.

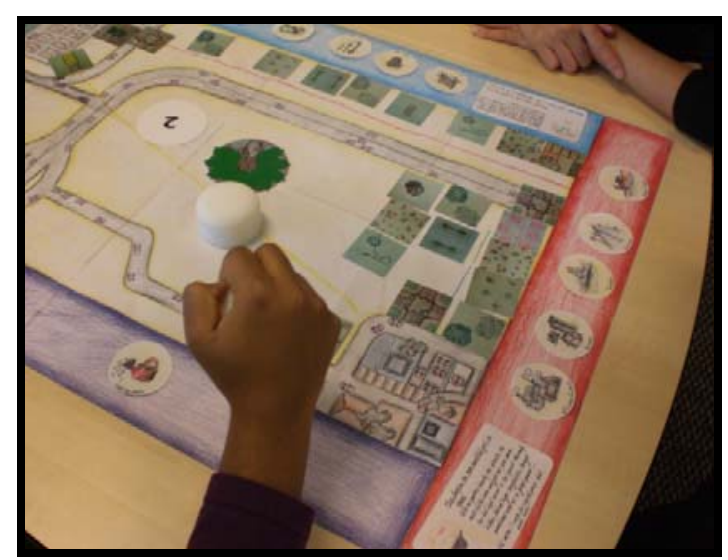

Figure 11: Paper Prototype Flourishing Future

\subsection{Technical Implementation}

Flourishing Future was implemented on the same digital tabletop as Totti. For Flourishing Future a model of the background system was first developed in excel, using visual basic, to make it easier to experiment with different values in this model.

\subsection{Methodology}

In this study the number of collaborative interactions was compared between one version of the game with and one version without cooperative gestures. Because the first study evaluation showed that measuring "how much children look at each other" is not relevant for a tabletop game, a different and more suitable way of evaluating was chosen. Two overt types of interactions were included in counting interactions; verbal and gestural interactions. These were divided into the following behavioral codes: proposals for action, reactions on these proposals, reasoning in general, confirmations, questions, general comments, exchange of tangible objects, grabbing a tangible object and placing one. This division is based on the guidelines provided by Morris et al (2006) and the evaluation of the first study. One interaction is defined as a verbal expression or gesture that is ongoing and is not stopped because of an interruption by someone else or because of a silence. Each interaction was scored as only one of these types. Exclamations, like 'yeah', were not scored because, in general, the other participants did not react on this and it is assumed that they did not influence cognitive processes. Besides, interactions that were caused by technical problems with the tabletop were not included because these problems varied among groups.

Webb (in: Dillenbourg et al, 1996) found that explanation is positively correlated with learning of the explainer. The categories 'proposals for action', 'reactions on these proposals' and 'reasoning in general' all seem to be linked to explanation. Therefore the sum of these three categories is compared between conditions. Dillenbourg et al (1996) argues that the sum of interactions of all the group members has to be compared between conditions, since the number of collaborative interactions is the result of a group process. To summarize, the sum of the categories 'proposals for action', 'reactions on these proposals' and 'reasoning in general' were compared on a group level, i.e. the indicated scores of all children in the group were summed up.

After the experiment the children filled out a questionnaire that focused on evaluating the cooperative gestures using by using the guidelines of Morris et al (2006). This evaluation gave an indication of how well the cooperative gestures were designed. Bad scores could lead to a bias in the research on collaborative interactions. For example: a child gets bored from doing the same cooperative gestures all the time and after a while stops collaborating. 
Ten groups of four children participated in the experiment. In total 40 children participated: 22 boys, 18 girls, all between 10 and 13 years old. These children were involved in a science club from different local middle schools. The children did not get any refund for their participation except for some drinks and snacks.

Each group played the game twice; once with the 'normal' version and once with the 'cooperative' version. The design was within-subjects. The two conditions were counterbalanced. Figure 12 shows children playing the game during the experiment. Figure 12a shows the 'cooperative' version; Figure 12b the 'normal' version.
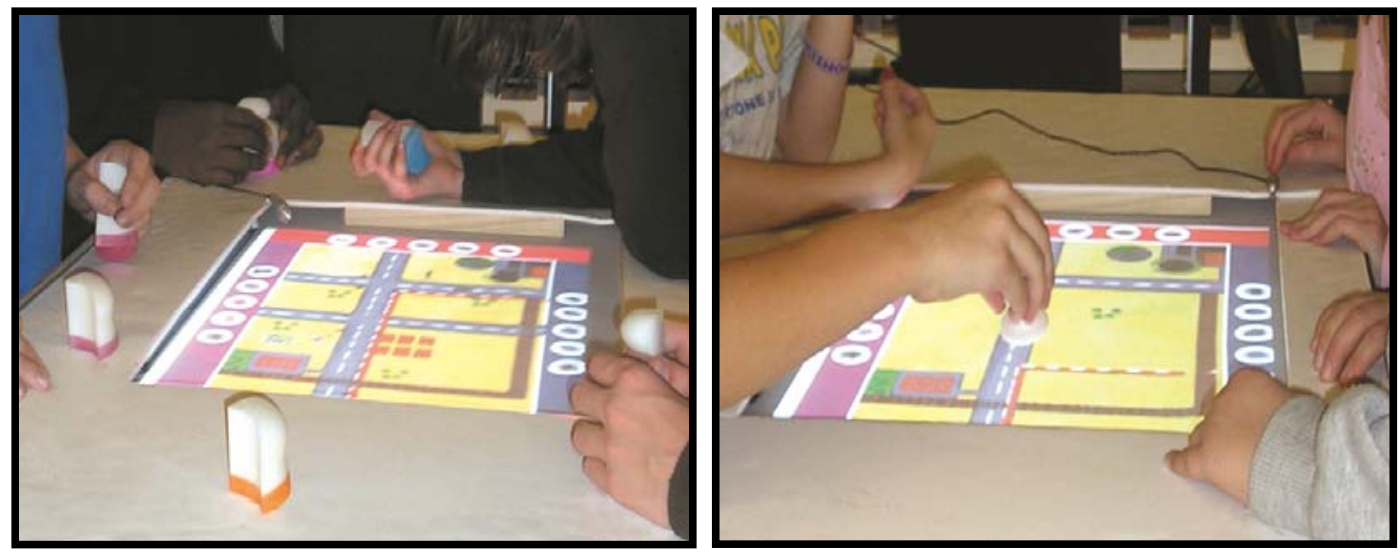

Figure 12: The left picture (12a) shows the children playing the version of the game with the cooperative gestures. The right picture (12b) shows the children playing the version of the game with the individual playing pieces.

As explained before, there were some problems with the implementation of the game. Therefore the computer vision system was simulated by the experimenter during game play. This was done by copying the children's actions on a separate computer.

At the start of the experiment a predefined introduction and the rules of the game were given by the experimenter. The children had the possibility to test the (combined) stamping and erasing once before starting the game. To limit the game duration, each group got 15 steps to reach the highest possible score. Within these 15 steps the children should strive for the highest possible level of environmental friendliness. Next, they played the game again but now in the other condition. Thus if they played with the 'normal' version first, they played with the 'cooperative' version the second time and vice versa. They still had the possibility to test the (combined) stamping and erasing once before the game started the second time.

Afterwards the children filled out a questionnaire that included questions on demographical data, their experience with the cooperative gestures and the experiment in general. The questions regarding the cooperative gesture were derived from the requirements for cooperative gestures in general (Jorda et al, 2005).

\subsection{Results Study 2: Cooperative Gestures}

The children performed more collaborative interactions in the game version including the cooperative gestures compared to the version with the 'normal' gestures. They also indicated that they liked the cooperative gestures.

Four sessions had problems with the audio taping; therefore only six sessions could be analyzed (see Table 1 ). The sums of the three categories - proposals for action, reactions on these proposals and reasoning in general - were calculated for each group and each condition and are displayed in Table 1 as 'explanation related interactions'. 'All interactions' in Table 1 is the sum of all the counted interactions per group per 
condition. It was decided not to include the data of group number 5 because these data were distorted by a girl not willing to talk.

Table 2: Quantitative Results of the second study; comparing two versions of the game.

* "Explanation related interaction" the interactions proposal, reaction and motivation

** In session 5 one of the participants refused to talk which distorted the data and therefore this session was not included.

\begin{tabular}{|l|l|l|l|l|}
\hline & \multicolumn{2}{|c|}{$\begin{array}{c}\text { Explanation related } \\
\text { interactions* }\end{array}$} & \multicolumn{2}{c|}{ All interactions } \\
\hline $\begin{array}{l}\text { Group } \\
\text { nr. }\end{array}$ & $\begin{array}{c}\text { Normal } \\
\text { condition }\end{array}$ & $\begin{array}{c}\text { Cooperative } \\
\text { condition }\end{array}$ & $\begin{array}{c}\text { Normal } \\
\text { condition }\end{array}$ & $\begin{array}{c}\text { Cooperative } \\
\text { condition }\end{array}$ \\
\hline 1 & 24 & 33 & 58 & 84 \\
\hline 2 & 105 & 121 & 189 & 208 \\
\hline 3 & 84 & 112 & 149 & 201 \\
\hline 4 & 64 & 80 & 127 & 160 \\
\hline $5 * *$ & 125 & 111 & 245 & 240 \\
\hline 6 & 87 & 116 & 203 & 257 \\
\hline total & 364 & 462 & 726 & 910 \\
\hline & & & & \\
\hline
\end{tabular}

The five sessions showed a statistically significant difference on the explanation collaborative between the two conditions in a two-sided paired samples t-test $(\mathrm{p}<0.01)$. As a side note, the total number of interactions was compared between the two conditions as well. This also led to a statistically significant result ( $\mathrm{p}<0.01)$. It can be concluded that the 'cooperative' version of the game increases the number of collaborative interactions over the 'normal' version. Some qualitative observations were done as well. Most children seemed to like the game and during the session the discussions about environmental friendliness seemed to get more depth. The results show that most children liked the second session better, while the conditions were counterbalanced. This could be explained by getting a better understanding of the game and its mechanisms in the second session.

Summarizing, this study introduces the educational game Flourishing Future. Two versions of this game, were developed: one with cooperative gestures and one without. These two versions were compared on the amount of collaborative interactions they evoked during game play. The results show that the 'cooperative' version evoked significantly more collaborative interactions. An increase in collaborative interactions improves the conditions for learning (Dillenbourg, 1991). Therefore it can be concluded that our 'cooperative' version of the game creates better conditions for learning compared to the 'normal' version..

\section{General Discussion}

The aim of our research was to increase the amount of collaborative interactions in a digital tabletop game to support collaborative learning. In the first study the researchers developed a multiplayer digital tabletop game which made use of degrees of collaboration to stimulate children to interact and participate in social interaction. The results showed an increase in the amount of collaborative interactions. In the second study the researchers used cooperative gestures to stimulate middle school children to collaborate and work together. Two versions with different sets of tools have been designed for this game, one normal version and one version consisting out of four pieces that should be put together to function. Results showed that when playing the cooperative version of the game the amount of collaborative interactions increased.

The children that participated in the evaluations were all members of a science club (STEMCELL), which could mean that they have an interest in science and this could influence the way they behave in a session and whether they liked the game. Being part of a science club could also mean that the children are more intelligent than other children from the same age.

Six parents of these children observed the session while their children were playing the game, the children did not know this, since they were out of sight. The parents thought that the game would be a good way to teach the children to work together and to perform tasks by discussing it with the others. 
We noticed that the children really liked playing the games. The children said things like "Oh! This is like a cool board game and a cool computer game!" or "Yeah!" when another leaf was added to the tree. Fun can be an important element overcoming the threshold to collaborate; it is motivating. Moreover, progressing in the game can be rewarding and can also be motivating to continue playing the game and collaborating. To increase the fun the children made additions to the rules during two sessions. For example, in one session the children made the rule that when one player needs something from another player, he had to do a dance. These exclamations and additions to the rules can influence the group behaviour in a positive way; the children motivate each other with their enthusiasm. Moreover, enthusiasm can lead to higher involvement of the children with the game, probably resulting in more collaborative interactions. Therefore, these exclamations and additions to the rules are important and interesting aspects of game experience.

Looking at the quantitative data of the Totti study, we can see that the criterion about exchanging of information was significant for level 2 to level 3, which can be explained by the set up of the game. During the exchange of information a lot of aspects of social interaction are combined (listening to another person, directing your attention to another person and responding to another person). In retrospect this is very important during the game, because this is essential for the tasks we incorporated in the social interaction levels. The two other criteria (person and action) were not significant. This can be explained by the tasks in the levels of social interaction. These levels asked for a certain amount of collaboration; the children needed to discuss plans and exchange objects. Specifically, in the second level the children had to discuss what to do and exchange objects to undertake actions, compelling them to socially interact. In the third level the children needed to combine their personal god objects to execute one single command, e.g. using cooperative gestures and thus collaborate. More exchange of information was required when finding a solution for a problem than when determining a path or exchanging objects. Therefore, in the difference between level 2 and 3, the criteria person and action were less present.

Moreover, we can state that measuring the looking at another person during tabletop games is less useful, because the attention is directed to the visual output of the tabletop. During "normal" collaboration people look at each other to confirm their direction of attention. During collaboration over a digital tabletop, people look at the table, but keep on doing other collaborative interactions.

Looking at the quantitative data of the Flourishing Future study the experiment shows a significant increase in the amount of collaborative interactions with the 'cooperative' version of the game compared to the 'normal' version. We do not understand exactly what causes this result. Is it that the children pay more attention to each other because everyone owns one piece of the object? Or do they feel they depend more on each other? From both studies results were shown to be significant, which means that we can say that using degrees of collaboration and cooperative gestures increases the amount of collaborative interactions.

Also, when looking at the technical feasibility of the two studies, we have learned to earlier check the integration of the hardware and the software. During the creation of the games the code has been constantly checked by using the provided simulator. The hardware was also checked by using simple codes to see whether all objects were registered. However, the two were never checked integrated until the evaluation. The problem of this integration probably lies in the highly visual nature of the game. Therefore we have learned to evaluate the integration of the software and the hardware along the process. Moreover, if feasible, the minimum amount of programs should be used for creating a game; the programs running on top of each other slowed down the communication from the infrared camera towards the processing computer via Java to Processing.

Moreover, we have learned that collaboration and thus also collaborative interactions are very personal. The amount of collaboration during a session depends on the total group; the people involved and there openness to work with others. In the fifth Flourishing Future session for example, one girl refused to talk; she was not open for collaboration and therefore the whole group mechanics changed. Therefore the amount of interactions varies a lot depending on participant's personalities, moods and willingness.

Referring to the use of traditional board games to encourage children to collaborate, we noted some advantages of digital tabletop games. A digital game can make use of rewards, visuals and changing 
themes. This is a benefit because this can keep children motivated and encouraged to play the game over and over again, while a traditional board game will never change.

In general cooperative interactions can be a beneficial means for stimulating social interaction and collaboration in other environments as well. The nature of cooperative gestures lays in the mutual understanding and execution of a command; this can be useful in working environments, schools, educational games and other educational environments. It can be used to execute specific actions together, but also to make decisions with a consensus of all the participants. Moreover, cooperative gestures implicitly persuade all participants to be involved and to create a mutual understanding of what is happening, simultaneously.

\section{Conclusions}

This paper describes the design of two tangible games on a digital tabletop with the goal to increase collaborative interactions among children in the Totti study and in the Flourishing Future study. In the Totti study three degrees of collaboration were incorporated in the game to increase the collaborative interactions needed. In the Flourishing Future study two versions of the game were implemented differing in interaction style: one requiring cooperative gestures and the other did not. The results show significant increases in collaborative interactions among normally developed children with both game designs. It can be concluded that designing digital tabletop games with tangible interaction according to the degrees of collaboration theory and cooperative gestures model can increase the number of collaborative interactions.

\section{Acknowledgements} -removed for anonymous reviewing-

\section{References}

Al Mahmud A, Mubin O, Octavia J R, Shahid S, Yeo L, Markopoulos P, Martens J (2007) aMAZEd: Designing an Affective Social Game for Children. In: Proceedings of the $6^{\text {th }}$ International Conference on Interaction Design and Children (IDC’07), 53-56.

Baker M, Hansen T, Joiner R, Traum D (1999) The role of grounding in collaborative learning tasks. In: P. Dillenbourg (Ed.), Collaborative learning: Cognitive and computational approaches, 31-63.

Bakker S, Vorstenbosch D, Hoven E. v.d., Hollemans G, Bergman T (2007) Weathergods: tangible interaction in a digital tabletop game. In: Proceedings of the $1^{\text {st }}$ Conference on Tangible and Embedded Interaction 2007(TEI’07), 151-152.

Barry M, Pitt I, (2006) Interaction Design: a Multidimensional Approach for Learners with Autism. In: Proceedings of the $5^{\text {th }}$ Conference on Interaction Design and Children (IDC’06), 33-36.

Bauminger N, Gal E, Goren-Bar D, (2007) Enhancing Social Communication in High Functioning Children with Autism through a Co-Located Interface. In: Proceedings of the International Workshop on Multimedia Signal Processing, 18-21.

Dillenbourg P (1991) What do you mean by collaborative learning? In: P. Dillenbourg (Ed) Collaborative-learning: Cognitive and Computational Approaches, 1-19.

Dillenbourg P, Baker M, Blaye A, O'Malley C (1996) The evolution of research on collaborative learning. In: Spada, E., and Reiman, P. (Ed) Learning in Humans and Machine: Towards an interdisciplinary learning science, 189-121.

Fitzmaurice GW, Ishii H, Buxton B, (1995) Bricks: Laying the Foundations for Tangible User Interfaces. In: Proceedings of Conference on Human Factors in Computing Systems (CHI’95), 442-449.

Fleck R, Rogers Y, Yuill N, Marshall P, Carr A, Rick J, Bonnett V (2009) Actions Speak Loudly with Words: Unpacking Collaboration Around the Table. In: Proceedings of Conference on Interactive Tabletops and Services (ITS’09), 189-196.

Gokhale AA (1995) Collaborative Learning Enhances Critical Thinking. J. Tech. Edu, 7, 22-30. 
Greenberg S, Marwood D, (2004) Real Time Groupware as a Distributed System: Concurrency control and its Effects on the Interface. In: Proceedings of European Conference on Computer Supported Cooperative Work (CSCW’04), 207-217.

Heijboer M, Hoven E v.d. (2008) Keeping up appearances: Interpretation of tangible artifact design. In: Proceedings of the Nordic Conference on Human Factors in Computing Systems (NordiCHI 2008), 162-171.

Hornecker E, Marshall P, Dalton NS, Rogers Y (2008) Collaboration and Interference: Awareness with Mice or Touch Input. In: Proceedings of the 2008 ACM conference on Computer supported cooperative work (CSCW’08), pp 167-176.

Jordà S, Kaltenbrunner M, Geiger G, Bencina R, (2005) The reacTable*. In: Proceedings of the International Computer Music Conference (ICMC’05).

Kahn KB, Mentzer JT, (1996) Logistics and interdepartmental integration. Int. J. Phys. Distr. and Log. Manag. DOI:10.1108/09600039610182753.

Kaltenbrunner M, Bencina R (2007) ReacTIVision: A Computer-Vision Framework for Table-Based Tangible Interaction. In: Proceedings of conference on Tangible and Embedded Interaction (TEI’07), 69-74.

Magerkurth C, Memisoglu M, Engelke T, Streitz NA, (2004) Towards the next generation of tabletop gaming experiences. In: Proceedings of Graphics Interface, 73-80.

Mandryk RL, Maranan DS, Inkpen KM (2002) False prophets: exploring hybrid board/video games. In: Extended Abstracts of CHI, 640-641

Mazalek A (2005) Media Tables: An extensible method fordeveloping multi-user media interaction platforms for shared spaces. Ph.D. thesis, Massachusetts Institute of Technology, Cambridge.

Morris MR, Huang A, Paepcke A, Winograd T(2006) Cooperative Gestures: Multi-User Gestural Interaction for Colocated Groupware. In: Proceedings of Human Factors in Computing Systems (CHI’06), 1201-1210.

Morris MR, Cassanego A, Piper AM, Huang A (2006) Mediating Group Dynamics through Tabletop Interface Design. In: Journal of IEEE computer graphics and applications, 65-73.

Morris MR, Ryall K, Shen C, Forlines C, Vernier F (2004) Beyond "Social Protocols": Multi-User Coordination Policies for Co-located Groupware. In: Proceedings of Computer Supported Cooperative Work (CSCW’04), 262-265.

Piper AM, O’Brien E, Morris MR, Winograd T (2006) SIDES: A Cooperative Tabletop Computer Game for Social Skills Development. In: Proceedings of Computer Supported Cooperative Work (CSCW’06), 1-10.

Real-Time strategy games, http://www.wikipedia.org/wiki/Real-time_strategy/, last accessed 7 February 2011.

Roschelle J, Teasley SD (1995) Construction of shared knowledge in collaborative problem solving. In: C. O'Malley (Ed.), Computer-supported collaborative learning. New York: Springer-Verlag.

Ryall K, Esenther A, Everitt K, Forlines C, (2005) iDwidgets: Parameterizing Widgets by User Identity. In: Proceedings of Interact, 1124-1128.

Scott SD, Grant KD, Mandryk RL (2003) System Guidelines for Co-located, Collaborative Work on a Tabletop Display. In: Proceedings of Conference on Computer Supported Cooperative Work (CSCW’03), 159-178.

Smith R, Hixon R, Horan B (1998) Supporting Flexible Roles in a Shared Space. In: Proceedings of conference on Computer Supported Cooperative Work (CSCW’98). DOI: 10.1145/289444.289494.

Stahl G, Koschmann T, Suthers D (2006) Computer-supported collaborative learning: An historical perspective. In: R. K. Sawyer (Ed.) Cambridge handbook of the learning sciences, 409-426.

Stewart J, Bederson BB, Druin A (1999) Single Display Groupware: a model for co-present collaboration. In: Proceedings of Human Factors in Computing Systems (CHI’99), 286-293. 
Suh NP (2006) Application of Axiomatic Design to Engineering Collaboration and Negotiation. In: Proceedings of The International Conference on Auditory Display (ICAD’06), 1-11.

Tse E, Greenberg S, Shen C (2006) Motivating Multimodal Interaction Around Digital Tabletops. In: (Video)Proceedings of the conference on Computer Supported Cooperative Work (CSCW'06), Video and two-page summary. Duration 3:25.

Ullmer B, Ishii H (2000) Emerging frameworks for tangible user interfaces. In: "Human-Computer Interaction in the New Millenium John M. Carroll, ed. 